\title{
Notational Comparison Analysis of Outdoor Badminton Men's Single and Double Matches
}

\author{
by \\ José Antonio Pérez-Turpin ${ }^{1}$, Carlos Elvira-Aranda ${ }^{1}$, David Cabello-Manrique ${ }^{2}$, \\ María José Gomis-Gomis ${ }^{1}$, Concepción Suárez-Llorca ${ }^{1}$,Eliseo Andreu-Cabrera ${ }^{1}$
}

The objectives of this study were to determine the results of the analysis of single and double outdoor badminton men's matches and to determine the relationship between technical and tactical aspects in a study organized by the BWF (Badminton Word Federation), on a sand surface. Twenty men's singles matches were recorded using video cameras and analysed with a Dartfish video analysis software package. Along with this, percentages of use of technical elements were analysed by comparing the different modalities. For the single format Lob, Clear, Drop, Smash, Drive were used, different from the double format that used Lob, Clear, Drop, Smash, Drive. The study confirmed the applicability of computerized notation analysis to determine the characteristics of Outdoor Badminton on sand.

Key words: outdoor badminton, notational analysis, technique, competition.

\section{Introduction}

Badminton appeared in Asia and Europe more than 2000 years ago (Tan et al., 2016). Badminton is a racket sport played by two or four players on a rectangular court (Lees, 2003; Tan et al., 2016), characterized by intermittent efforts of high intensity and short duration (Abián-Vicén et al., 2012; Cabello and Gonzalez-Badillo, 2003; Valldecabres et al., 2017).

Technical variables have been studied in professional badminton (Cabello and GonzálezBadillo, 2003; Faude et al., 2007; Leong and Krasilshchikov, 2016; Zhang et al., 2013), and its analysis allows to identify the most decisive actions in this sport (Valldecabres et al., 2017). Researchers have studied the stroke average (Abián et al., 2014; Laffaye et al., 2015; Ming et al., 2008), stroke frequency (Alcock and Cable, 2009; Ming et al., 2008) and types of strokes used (Chiminazzo et al., 2018; Laffaye et al., 2015). On the other hand, match performance has been examined using time variables (Phomsoupha and Laffaye, 2015) as it has been shown that the results in professional badminton depend on game time, effective game time, set time, rally time and rest time between rallies (Abián-Vicén et al., 2018; Chiminnazzo et al., 2018; Ming et al., 2008; Phomsoupha and Laffaye, 2015; Laffaye et al., 2015; Valldecabres et al., 2017). It should be noted, however, that most current studies relate to indoor badminton games and for this reason it is hard to find studies that analyze technical and timing characteristics in outdoor badminton.

Mixed methods combine qualitative and quantitative elements as research strategies (Johnson et al., 2004; Teddlie and Tashakkori, 2003). They have been used in sports such as basketball, soccer, fencing and judo (Camerino et al., 2012; DeSchiver, 2007; Iglesias and Angular, 2012; Readdy et al., 2014; Sarmento et al., 2014). Within this type of research we find the observational methodology (Anguera, 1979) which allows to collect the data directly from the participants due to an accessible method such as recording (Anguera and Hernández-Mendo, 2014). Video analysis techniques can help 
understand the game itself, as they allow to evaluate the actions during the competition. These techniques are very important in most racket sports (Abian-Vicen et al., 2013). Notational analysis is one of the methods that can be applied within the observational methodology to determine the characteristics of a badminton game (Hughes et al., 2007) and research exists that uses it to investigate these characteristics in this sport (Abdullahi and Coetzee, 2017).

The objective of this study was to analyze and compare technical and timing variables in professional players between the individual and double modalities in outdoor badminton.

\section{Methods}

\section{Participants}

Male individual matches $(\mathrm{n}=38)$ analyzed were played by 8 professional players (age $33 \pm 6.5$ years, body height $181.5 \pm 3.5 \mathrm{~cm}$ and body mass $70.3 \pm 8.6 \mathrm{~kg}$ ) of the Spanish National Badminton Team. They were played in the 1 vs. $1(n=20)$ and 2 vs. $2(n=18)$ modalities on a sand surface. All matches were played according to the official badminton rules.

The recording process did not affect the spontaneous behavior of players/teams due to it being non-invasive and a common way to monitor competitions. The study was approved by the Bioethics Commission of the University of Alicante, and complied with the ethical principles stated by the Declaration of Helsinki.

Design

An observational design was used with a notational analysis, technical variables were analyzed and organized by the BWF (Badminton Word Federation). The registered technical variables were (Chiminazzo et al., 2018):

Serve - when a player touches the shuttle at the beginning of the point,

Lob - a shot towards the back of the opponent's court with a raining trajectory (Phomsoupha and Laffaye, 2015),

Net drop - a precise shot near the net, which includes the push, kill and brush (Abian-Vicen et al., 2012),

Smash - an aggressive overhead shot with a downward trajectory (Phomsoupha and Laffaye, 2015),

Clear - an overhead shot to the back of the court in a rising trajectory of the shuttle (Abian-Vicen et al., 2012),
Drive - a powerful shot made at the middle body height from the middle of the court and which has a flat trajectory (Phomsoupha and Laffaye, 2015).

\section{Procedures}

The footage was shot with two cameras Sony DCR-cx 280 with a focal length of 29.8 - 953.6 $\mathrm{mm}$ and a resolution of $1920 \times 1080 \mathrm{p} / 50 \mathrm{fps}$. The cameras were placed at the bottom of the court. The court $(13.40 \times 6.10 \mathrm{~m})$ was registered in its entirety to ensure a complete view of all the actions. Variables were analyzed with a Dartfish 9.0 program (Guo, 2018) by two observers graduated in Sports Science with two years of experience in performance analysis.

Both observers completed specific training of the actions to register. The intra-observer reliability was calculated in both observers using the mathematical formula of Hughes (2004):

$$
\operatorname{Erm}(\%)=(\Sigma(\bmod [\mathrm{V} 1-\mathrm{V} 2]) / \text { Vaverage }) *
$$

where V1 are the frequencies of the first operator display and V2 the frequencies of the second operator, Vaverage displays the average of the visualization frequencies and mod is the module.

The reliability on the intra-observer analysis obtained a margin for error of less than 5\%, reaching acceptable margins for error in the display and analysis (James et al., 2007). For the inter-observer analysis, Kappa calculations were carried out using SPPS software (v.18.0), reaching an inter-observer concordance value of 0.95 (Landis and Koch, 1977).

\section{Statistical analysis}

Descriptive (mean and standard deviation) and inferential tests were performed using SPPS software (v.18.0). The Kolmogorov-Smirnov test was used to analyze normality of data. The Mann Whitney U procedure with a post hoc Bonferroni test $(p<.01)$ was applied to analyze variables with non-parametric distributions. Variables with parametric distributions were analyzed with using the T-Student test $(p<.05)$.

\section{Results}

Figure 1 shows the stroke distribution used in 1 vs. 1 and 2 vs. 2 modalities. The lob ( $\mathrm{Z}=-5.097$; $p=.001)$ and drive $(Z=-3.133 ; p=.002)$ were most used in 1 vs. $1(25 \%$ and $23 \%)$ and in 2 vs. $2(22 \%$ and $24 \%$ ). We found significant differences 
between groups in the use of the smash and clear $(\mathrm{p}<.05)$.

Figure 2 shows the average frequency of the type of the service used in each modality. A short serve presented a greater use in both modalities ( $p$ $<.05)$. A deep serve was more used in 2 vs. 2, while in 1 vs. 1 the half court serve was recurrent. No significant differences were found between modalities.

Net errors committed with the highest proportion may be observed in Figure 3. The smash $(38 \%$ and $34 \%)$, drive $(29 \%$ and $31 \%)$ and net drop $(22 \%$ and $19 \%)$ were most frequent $(p<$. 05 ) in 1 vs. 1 and 2 vs. 2 modalities. We did not find any significant differences between particular modalities.

Figure 4 shows the efficiency in the last stroke of the rally. The most used strokes to end were the smash, drive and lob in 1 vs. 1 modality. In 2 vs. 2 modality, it was the smash that was most frequently used. The greater proportion of effectiveness of strokes in 1 vs. 1 was found in the serve $(58 \%)$, smash $(83 \%)$ and drop (71\%). In 2 vs. 2 modality they were the serve (61\%), smash $(66 \%)$ and net drop (56\%).

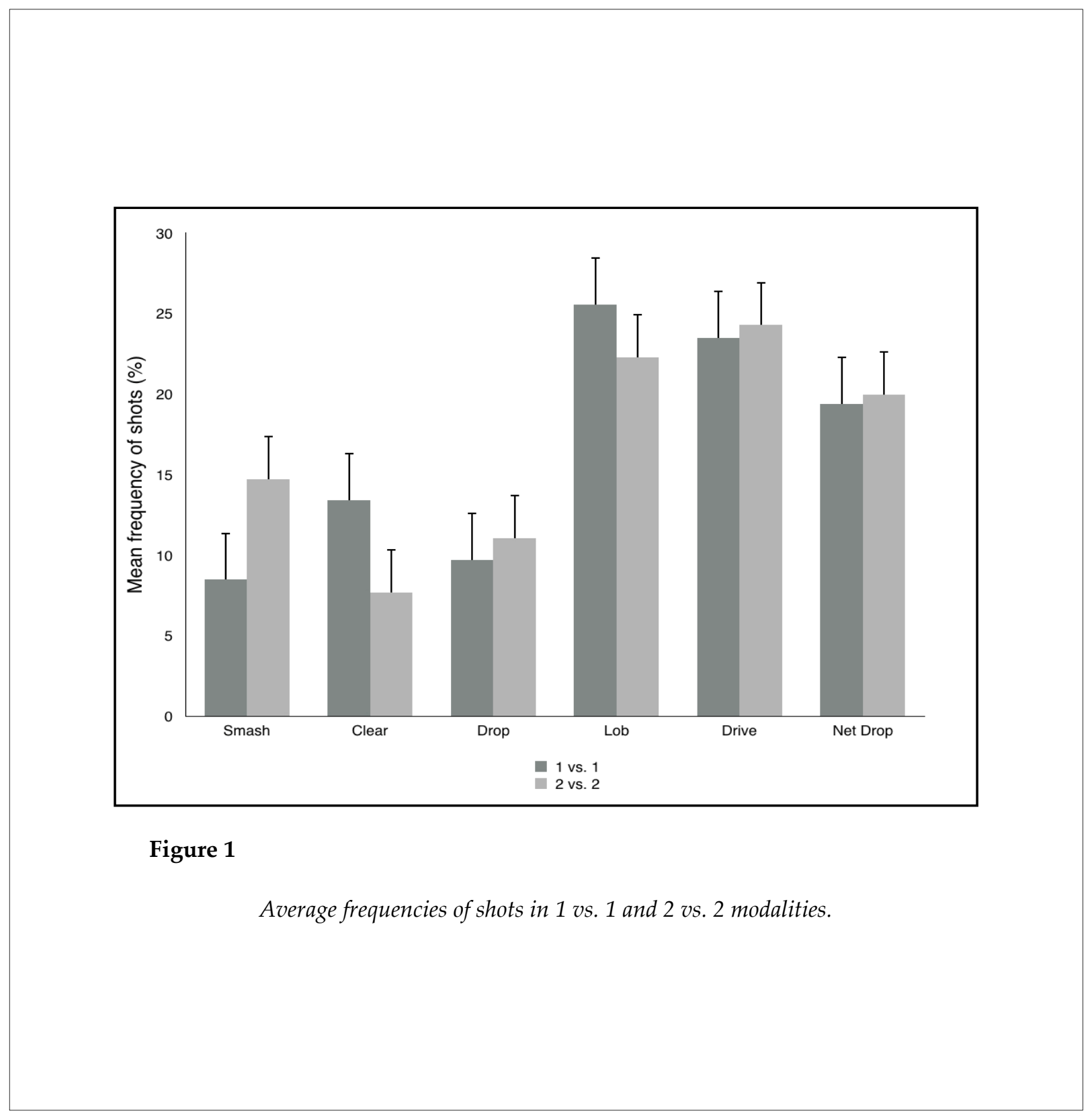




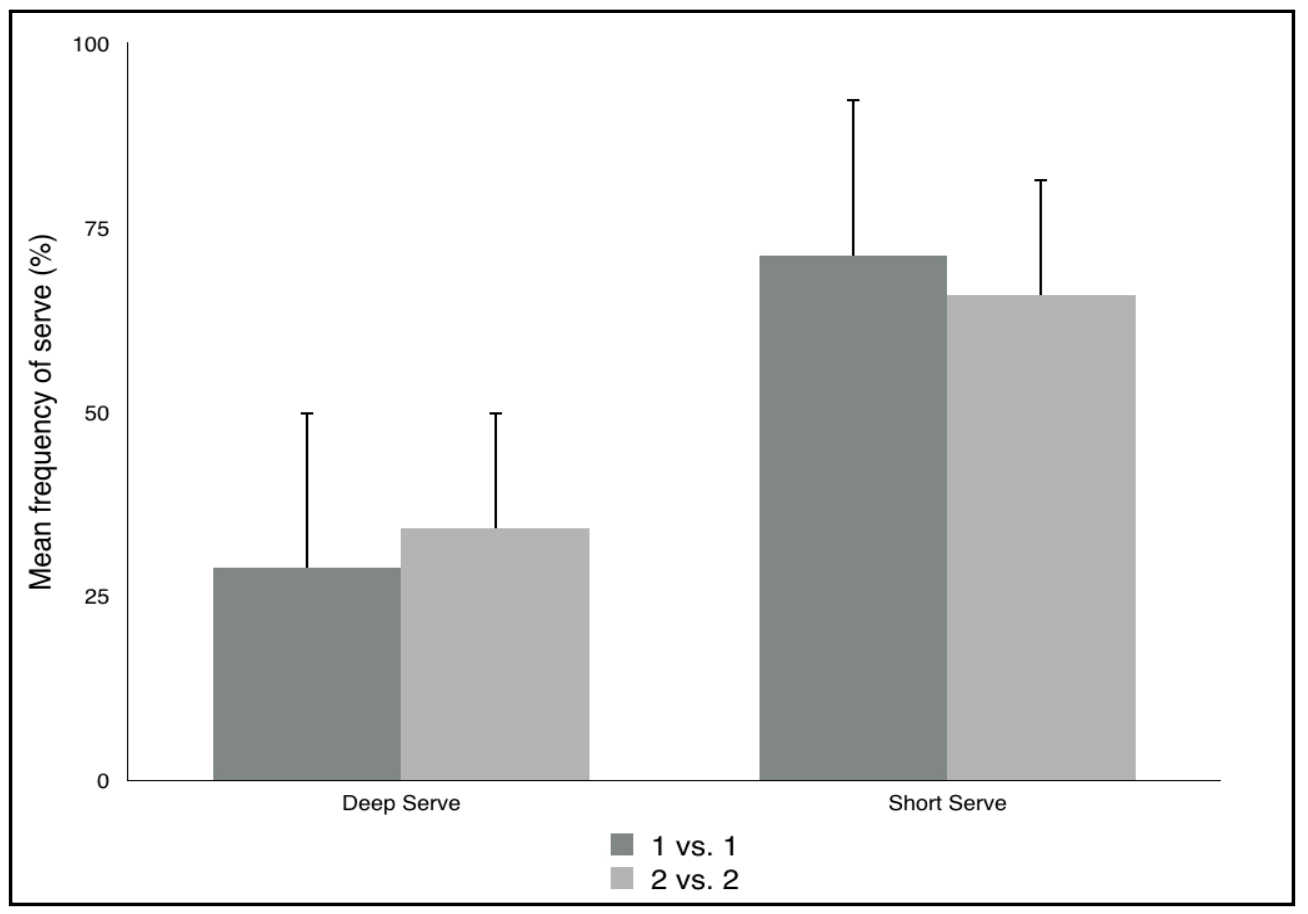

Figure 2

Average frequencies of the serve's type in 1 vs. 1 and 2 vs. 2 modalities.

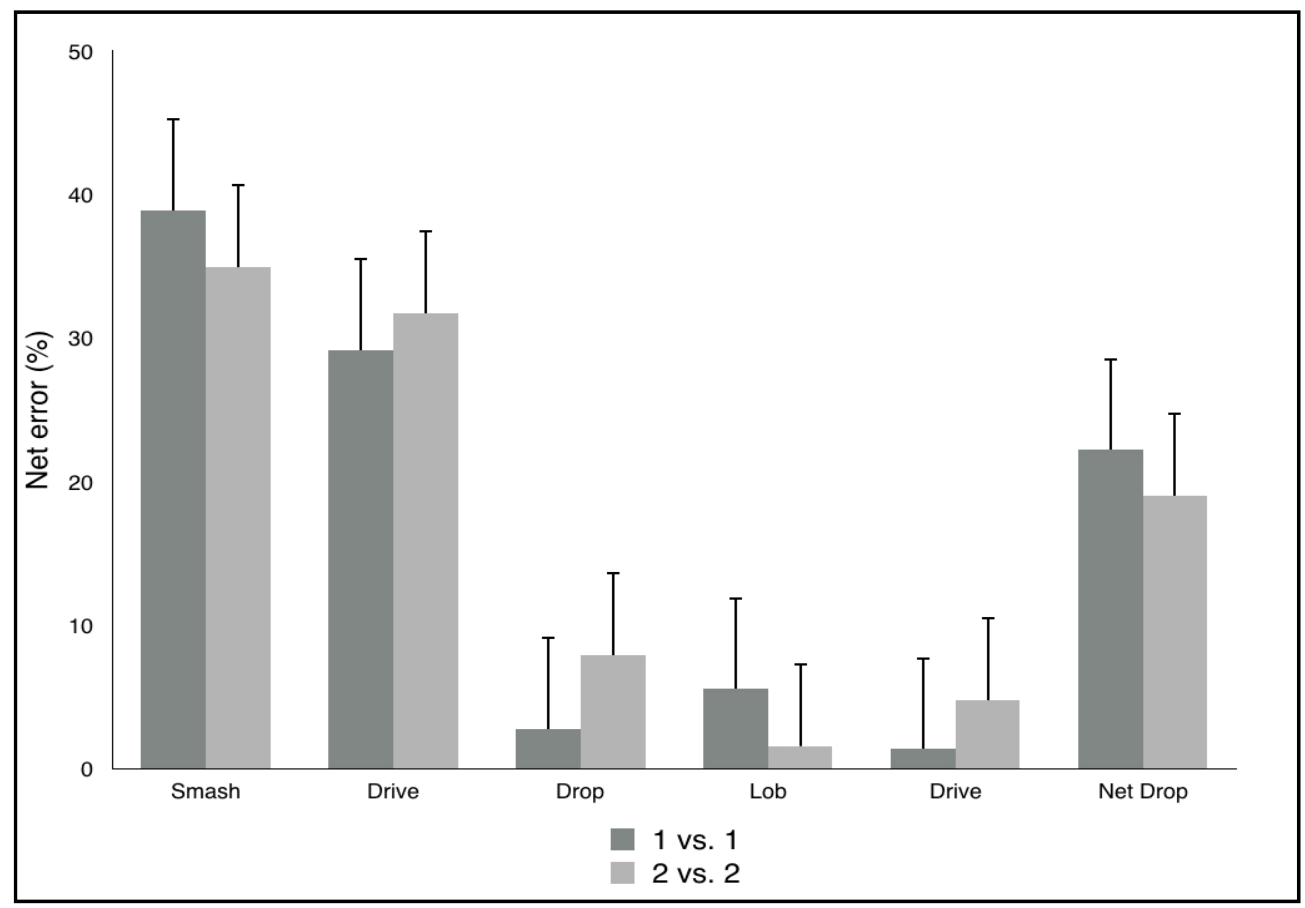

Figure 3

Net error in 1 vs. 1 and 2 vs. 2 modalities. 


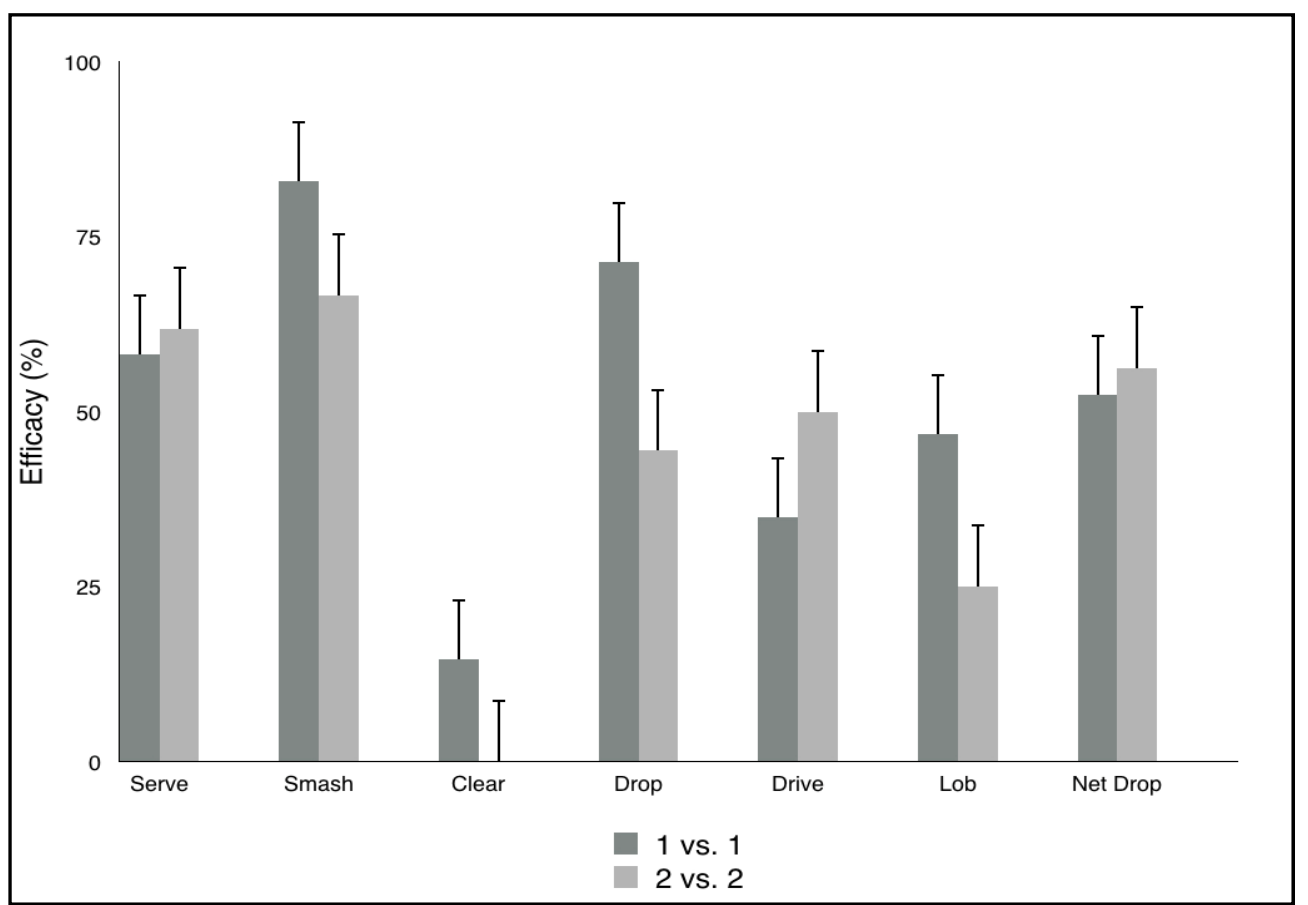

Figure 4

Efficacy of the shot's type in 1 vs. 1 and 2 vs. 2 modalities.

\section{Discussion}

The objective of this study was to analyze and compare technical variables between the individual and double modalities in outdoor badminton in professional players. There are no previous studies that have analyze technical variables in outdoor badminton and played on sand, thus any comparison made will be with the Olympic modality. Notational analysis on technical variables has been used in a multitude of studies with different objectives within the game, different levels of competition and specific aspects of the game (Abian-Vicen et al., 2012; Abián et al., 2014; Ming et al., 2008).

In our study, the most used strokes in 1 vs. 1 and 2 vs. 2 modalities were the lob (25\% and 23\%, respectively) and drive $(22 \%$ and $24 \%$, respectively). Chiminazzo et al. (2018) showed that the most frequent hits were the lob, drop, smash, clear and drive respectively. Ming et al. (2008) also found that the lob was the most used hit in badminton players. Abdullahi and Coetzee (2017) presented the drive as a stroke more frequently used per game in the African Badminton Championship. Tong and Hong (2000) showed the lob, smash, net drop and clear as the most frequently used strokes. Pearce (2002) also recorded as preferred strokes the lob, clear and drop. The use of strokes used on sand did not differ from those found in traditional match situations.

For the serve, we found that a short serve was more frequent than a deep serve in both modalities. Valldecabres et al. (2015) showed similar results indicating a short serve as more common, while reflecting that a deep serve was an infrequent stroke. The type of a serve used may be influenced mainly by the number of players and 
not so much by the area used.

The most frequently encountered net errors were the smash (38\% and $34 \%)$, drive ( $29 \%$ and $31 \%$ ) and net drop (22\% and $19 \%$ ), in both 1 vs. 1 and 2 vs. 2 modalities. Abian-Vicen et al. (2013) showed the smash and net drop in men and women as strokes that resulted in most common unforced errors.

Among the registered strokes that were most used to end a rally were the smash and drive (Abian-Vicen et al., 2013). Chen et al. (2011) also showed that the smash was the preferred stroke to win points. Our results do not differ from those as they indicate the smash as the last most recorded stroke. As with the serve, the use of a type of a stroke to finish was due to the action itself or the number of players and not related to the surface.

The fundamental characteristic of this study is the type of surface used to play. Exercise on a sand surface requires physiological and biomechanical mechanisms different from other surfaces (Pinnington and Dawson, 2001; Pinnington et al., 2005). The energy expenditure is greater due to an increase in kinematic patterns and muscle activation, in addition to a reduction in muscle-tendon efficiency (Zamparo et al., 1992). In the same way, a sand surface modifies stride length and increases the race cadence (Pinnington et al., 2005). Surface change can vary technical variables used with respect to those studied in indoor competitions, but, as we have suggested previously, the action itself and the number of players can influence the type of a stroke used to a greater degree.

\section{Conclusions}

The study shows technical characteristics of outdoor badminton on a sand surface. Registers of the variables do not show significant differences with matches played on the official surface. Players adapt to the new surface, and the number of participants along with the moment of the game influence the type of a stroke used to a greater degree, although it would also be necessary to study the importance of wind on the game in outdoor badminton. More studies are needed with a larger sample and register of variables to observe the impact of outdoor conditions on the game.

\section{References}

Abdullahi Y, Coetzee B. Notational singles match analysis of male badminton players who participated in the African Badminton Championships. Int J Perf Anal Spor, 2017; 17: 1-16

Abián-Vicén J, Coso J, González-Millán C, Salinero JJ, Abián P. Analysis of dehydration and strength in elite badminton players. PLoS One, 2012; 7(5): e37821

Abián P, Castanedo A, Feng XQ, Sampedro J, Abian-Vicen J. Notational comparison of men's singles badminton matches between Olympic Games in Beijing and London. Int J Perf Anal Spor, 2014; 14(1): 4253

Abián-Vicén J, Sanchez L, Abián P. Performance structure analysis of the men's and women's badminton doubles matches in the Olympic Games from 2008 to 2016 during playoffs stage. Int J Perf Anal Spor, 2018; 18: 633-644

Alcock A, Cable NT. A comparison of singles and doubles badminton: Heart rate response, player profiles and game characteristics. Int J Perf Anal Spor, 2009; 9(2): 228-237

Anguera MT. Observational typology. Quality \& quantity. European-American Journal of Methodology, 1979; 13(6): 449-484

Anguera MT, Camerino O, Castañer M, Sanchez Algarra P. Mixed methods in research into physical activity and sport. Rev Psicol Deporte, 2014; 23(1): 123-130

Anguera MT, Hernandez-Mendo A. Observational methodology and sports psychology: state of affairs. Rev Psicol Deporte, 2014; 23(1): 103-109

Binnie MJ, Dawson B, Pinnington H, Landers G, Peeling P. Sand training: a review of current research and practical applications. J Sport Sci, 2014; 32(1): 8-15

Cabello Manrique D, González-Badillo JJ. Analysis of the characteristics of competitive badminton. Brit J Sport Med, 2003; 37(1): 62-66

Camerino O, Iglesias X, Gutiérrez A, Prieto I, Campaniço J, Angular MT. Optimizing techniques and dynamics in individual sports. Abdingdon, UK: Routledge, 82-116; 2012

Chen HL, Chen WUTC. Physiological and notational comparison of new and old scoring systems of singles matches in men's badminton. Asian Journal of Physical Education and Recreation, 2011; 17(1): 6-17 
Chiminazzo JGC, Barreira J, Luz LS, Saraiva WC, Cayres JT. Technical and timing characteristics of badminton men's single: comparison between groups and play-offs stages in 2016 Rio Olympic Games. Int J Perf Anal Spor, 2018; 18: 245-254

DeSchiver TD. Much adieu about Freddy: Freddy Adu and attendance inn Major League Soccer. Journal of Sport Management, 2007; 21(3): 438-451

Faude O, Meyer T, Rosenberger F, Fries M, Huber G, Kindermann W. Physiological characteristics of badminton match play. Eur J Appl Physiol, 2007; 100(4): 479-485

Guo WG. Application of video feedback system in analysis and diagnosis of Wushu routine movement. Journal of Interdisciplinary Mathematics, 2018; 21(5): 1187-1991

Hughes M. Notational analysis - A mathematical perspective. Int J Perf Anal Spor, 2004; 4(2): 97-139

Hughes MD, Hughes MT Behan H. The evolution of computerized notational analysis through the example of racket sports. International Journal of Sports Science and Engineering, 2007; 1(1): 3-28

James N, Taylor J, Stanley S. Reliability procedures for categorical data in Performance Analysis. Int J Perf Anal Spor, 2007; 7(1): 1-11

Johnson R, Onwuegbuzie A. Mixed method research: A research paradigm whose time hascome. Educ Res, 2004; 33: 14-26

Laffaye G, Phomsoupha M, Dor F. Changes in the game characteristics of a badminton match: A longitudinal study through the Olympic game finals analysis in Men's singles. J Sport Sci Med, 2015; 14(3): 584

Landis JR, Koch GG. The measurement of observer agreement for categorical data. Biometrics, 1977; 33: 159174

Lees A. Science and the major racket sports: A review. J Sport Sci, 2003; 21(9): 707-732

Leong KL, Krasilshchikov O. Match and game performance structure variables in elite and youth International badminton players. Journal of Physical Education and Sport, 2016; 16(2): 330-334

Ming CL, Keong CC, Ghosh AK. Time motion and notational analysis of 21 point and 15 point badminton match play. International Journal of Sports Science and Engineering, 2008; 2(4): 216-222

Pearce A. A physiological and notational comparison of the conventional and new scoring systems in badminton. Journal of Human Movement Studies, 2002; 43(1): 49-67

Phomsoupha M, Laffaye G. The science of badminton: Game characteristics, anthropometry, physiology, visual fitness and biomechanics. Sports Med, 2015; 45(4): 473-495

Pinnington HC, Dawson B. The energy cost of running on grass compared to soft dry beach sand. J J Sci Med Sport, 2001; 4: 416-430

Pinnington HC, Lloyd DG, Besier TF, Dawon B. Kinematic and electromyography analysis of sub maximal differences running on a firm surface compared with soft, dry sand. Eur J Appl Physiol, 2005; 94: 242-253

Readdy T, Raabe J, Harding J. Student-Athletes' perceptions of an extrinsic reward program: a mixed-methods exploration of self-determination theory in the context of college football. Journal of Applied Sport Psychology, 2014; 26: 157-171

Sarmento H, Anguera MT, Pereira A, Marques A, Campaniço J, Leitão J. Patterns of play in the counterattack of elite football teams - A mixed method approach. Int J Perf Anal Spor, 2014; 14(2): 411-427

Teddlie C, Tashakkori A. Major issues and controversies in the use of mixed methods in the social and behavioral sciences. Thousand Oaks, CA: Sage, 3-50; 2003

Valldecabres R, Benito AM, Casal C, Pablos C. 2015 Badminton World Championship: Singles final men's vs women's behaviours. Journal of Human Sport and Exercise, 2017; 12(3proc): 5775-5788

Zamparo P, Perini R, Orizio C, Sacher M, Ferretti G. The energy cost of walking or running on sand. Eur J Appl Physiol, 1992; 65: 183-187

Zhang B, Li F, Jiang W. Mixed doubles match technical and tactical analysis of world badminton champion based on mathematical statistics. Advances in Physical Educational, 2013; 3(4): 154-157

\section{Corresponding author:}

\section{David Cabello-Manrique}

Department of Physical Education and Sport,

University of Granada, Granada, Spain.

E-mail: dcabello@ugr.es 